OPEN ACCESS

Edited by:

Yin $L i$,

Rutgers, The State University of New Jersey, United States

Reviewed by:

Nelson Garcia

University of Minnesota Twin Cities,

United States

Zhaorong $\mathrm{Hu}$,

China Agricultural University, China

Lingli Dong,

Institute of Genetics

and Developmental Biology (CAS),

China

*Correspondence:

Shengbao Xu

xushb@nwsuaf.edu.cn

Xiaoming Wang

wangxm@nwsuaf.edu.cn

Specialty section:

This article was submitted to Evolutionary and Population Genetics,

a section of the journal

Frontiers in Genetics

Received: 30 June 2020 Accepted: 29 October 2020 Published: 27 November 2020

Citation:

Lu Y, Zhao P, Zhang A, Ma L, Xu S and Wang $X$ (2020) Alternative

Splicing Diversified the Heat Response and Evolutionary Strategy of Conserved Heat Shock Protein 90s in Hexaploid Wheat (Triticum aestivum

L.). Front. Genet. 11:577897.

doi: 10.3389/fgene.2020.577897

\section{Alternative Splicing Diversified the Heat Response and Evolutionary Strategy of Conserved Heat Shock Protein 90s in Hexaploid Wheat (Triticum aestivum L.)}

\author{
Yunze Lu',2, Peng Zhao' ${ }^{1}$, Aihua Zhang ${ }^{1}$, Lingjian $\mathrm{Ma}^{1}$, Shengbao $\mathrm{Xu}^{1 *}$ and \\ Xiaoming Wang ${ }^{1 *}$
}

${ }^{1}$ State Key Laboratory of Crop Stress Biology for Arid Areas, College of Agronomy, Northwest A\&F University, Yangling, China, ${ }^{2}$ School of Landscape and Ecological Engineering, Hebei University of Engineering, Handan, China

Crops are challenged by the increasing high temperature. Heat shock protein 90 (HSP90), a molecular chaperone, plays a critical role in the heat response in plants. However, the evolutionary conservation and divergence of HSP90s homeologs in polyploidy crops are largely unknown. Using the newly released hexaploid wheat reference sequence, we identified 18 TaHSP90s that are evenly distributed as homeologous genes among three wheat subgenomes, and were highly conserved in terms of sequence identity and gene structure among homeologs. Intensive time-course transcriptomes showed uniform expression and transcriptional response profiles among the three TaHSP90 homeologs. Based on the comprehensive isoforms generated by combining full-length single-molecule sequencing and Illumina short read sequencing, 126 isoforms, including 90 newly identified isoforms of TaHSP90s, were identified, and each TaHSP9O generated one to three major isoforms. Intriguingly, the numbers and the splicing modes of the major isoforms generated by three TaHSP9O homeologs were obviously different. Furthermore, the quantified expression profiles of the major isoforms generated by three TaHSP90 homeologs are also distinctly varied, exhibiting differential alternative splicing (AS) responses of homeologs. Our results showed that the AS diversified the heat response of the conserved TaHSP90s and provided a new perspective for understanding about functional conservation and divergence of homologous genes.

Keywords: wheat, heat stress, HSP90, alternative splicing, evolutionary conservation and divergence

\section{INTRODUCTION}

As a result of global warming and increasing frequent short episodes of extreme high temperature (Liu et al., 2014; Prasad and Djanaguiraman, 2014), heat stress has become one of the major factors that limit crop production and quality (Asseng et al., 2015; Lesk et al., 2016; Zhao et al., 2017). Each degree Celsius increase in global mean temperature would reduce crop yield by $6-10 \%$ under heat stress (Lesk et al., 2016; Zhao et al., 2017). 
Plants have evolved complex systems to cope with heat stress. Heat shock proteins (HSPs), which function as molecular chaperones, have well-established roles in the heat response (Wang et al., 2004). Ninety kDa HSPs (HSP90s) function not only in protein folding, degradation, and transportation, as characteristic of chaperones, but also in signaling transduction and protein kinase activity regulation, in an ATP-dependent manner (Carretero-Paulet et al., 2013; Schopf et al., 2017). Under heat stress, the 70-kDa HSP/HSP90 complex disassociates and releases the master heat response regulator, heat shock transcription factor A1s (HSFA1s), resulting in the activation of the plant heat-responsive transcription cascade (Hahn et al., 2011; Ohama et al., 2017). More recently, HSP90s have been proven to have the ability to stabilize the circadian clock ZEITLUPE and auxin cofactor F-box protein to maintain plant growth under heat stress (Wang et al., 2016; Gil et al., 2017). Under normal conditions, the downregulated expression of Arabidopsis HSP90s results in abnormal growth and development, such as embryo defects (Queitsch et al., 2002). HSP90s are also considered to exert capacitor-buffering effects of genetic perturbation, such as genetic variations and mutations, on plant morphology and phenotype (Rutherford and Lindquist, 1998; Geiler-Samerotte et al., 2016).

The identification and expression pattern analysis of HSP90s have been widely reported in Arabidopsis, rice, chickpea, and pigeonpea (Krishna and Gloor, 2001; Swindell et al., 2007; Hu et al., 2009; Agarwal et al., 2016). In Arabidopsis, seven AtHSP90s were characterized and classified into different subfamilies based on their subcellular localization. Under normal condition, HSP90AA (AtHSP90-1) and HSP90ABs (AtHSP902-AtHSP90-4) are located in the cytoplasm, and HSP90C1 (AtHSP90-5), HSP90C2 (AtHSP90-6), and HSP90B (AtHSP907) are located in chloroplasts, mitochondria, and endoplasmic reticulum, respectively. Under heat stress, HSP90s were found to translocate into the nucleus, to regulate the expression of heat stress-responsive genes (Meiri and Breiman, 2009; Wang et al., 2016). The HSP90AAs are highly heat inducible, whereas HSP90ABs are constitutively expressed in Arabidopsis and rice (Yabe et al., 1994; Swindell et al., 2007; Hu et al., 2009). Similar heat response patterns of HSP90s within a subfamily are observed in Populus and allotetraploid tobacco (Zhang et al., 2013; Song et al., 2019). Meanwhile, previous studies also demonstrate the conservation in terms of exon-intron structures and protein motifs of HSP90s within a subfamily during plant evolution (Chen et al., 2006; Xu et al., 2012; Zhang et al., 2017).

Hexaploid wheat (Triticum aestivum L.), a major crop worldwide, contains three subgenomes, AA, BB, DD. These three subgenomes derive from three diploids through two major hybridization events. The first hybridization occurs between the diploid T. urartu (AA progenitor) and an unknown diploid Aegilops species (BB progenitor), possibly Aegilops sharonensis or Ae. speltoides, leading to the emergence of allotetraploid wild emmer wheat (T. turgidum ssp. Dicoccoides, AABB). The wild emmer wheat subsequently evolves into durum wheat (T. turgidum ssp. Durum, AABB). The second hybridization between tetraploid emmer wheat and the diploid Ae. tauschii (DD progenitor) results in the hexaploid wheat (AABBDD)
(Dubcovsky and Dvorak, 2007; Marcussen et al., 2014). Due to the short polyploidy history, the hexaploid wheat is used as a model in the field of polyploidy evolution study, and the functional conservation and divergence of the homeologous genes in hexaploid wheat have always been intriguing (Borrill et al., 2015; Ramírez-González et al., 2018). At transcriptional level, expression partitioning of wheat homeologous genes is commonly observed, and the ratio of partially expressed genes ranges from about $55 \%$ in normal condition to around $68 \%$ in stress condition such as heat, drought, and salt (Leach et al., 2014; Liu et al., 2015; Zhang et al., 2016). Thus, expression partitioning of homeologous genes is thought to be a common strategy for abiotic stress acclimation in hexaploid wheat (Liu et al., 2015; Zhang et al., 2016).

In hexaploid wheat, nine cytosolic TaHSP90s have been reported, of which three TaHSP90AAs are highly expressed in the reproductive organs that are necessary for seedling growth, while six TaHSP90ABs are constitutively expressed that are essential for disease resistance (Wang et al., 2011). However, the evolution process of HSP90s during wheat polyploidization and the functional conversation and divergence between TaHSP9O homeologs in hexaploid wheat remain largely limited.

With posttranscriptional regulation, many heat response genes, including several types of transcription factors and HSPs, change their alternative splicing (AS) patterns and generate new isoforms, expanding the diversity of proteome and regulation modes in the heat response (Jiang et al., 2017; Keller et al., 2017; Liu et al., 2018). For example, under normal conditions, HSFA2 mainly encodes a truncated isoform without transcriptional activation activity, while under heat stress, the intact and transcriptionally active isoform is largely expressed to induce the expression of heat response genes (Sugio et al., 2009; Cheng et al., 2015). Interestingly, a new, small truncated form is induced, and this results in the autoregulation of HSFA2 under severe heat stress (Liu et al., 2013). Whether and how HSP90s respond to heat stress with posttranscriptional regulation remains to be determined. If it is, what posttranscriptional regulation means for HSP90s is intriguing. The advantages of full-length singlemolecule sequencing with long sequencing read provide powerful tool for accurate isoform detection and could be suitable to answer this question.

In this study, we performed a genome-wide identification of the TaHSP90s in hexaploid wheat using the newly released wheat reference sequence (IWGSC RefSeq v1.0) (International Wheat Genome Sequencing Consortium [IWGSC], 2018) and investigated the transcriptional and AS reprogramming of TaHSP90s using the dynamic and intensive heat response transcriptomes generated by combining full-length singlemolecule sequencing and Illumina short read sequencing in our previous study (Wang et al., 2019). The 18 TaHSP90s were highly conserved in terms of sequence and transcriptional response pattern among TaHSP90 homeologs, while the number, the splicing modes, and the AS responses of the major isoforms generated by TaHSP90s homeologs were distinctly different. Our findings indicated that AS regulation diversified the heat response of the conserved TaHSP 90 homeologs and possibly facilitated the evolutionary divergence of TaHSP90 homeologs. 


\section{MATERIALS AND METHODS}

\section{Identification of HSP90s in Hexaploid Wheat and Its Progenitors}

Genome and protein sequences and genome annotation files of hexaploid wheat were downloaded from $\mathrm{URGI}^{1}$, and the protein sequences of Ae. tauschii, T. urartu, durum wheat, and wild emmer wheat were downloaded from the EnsemblPlants database $^{2}$. Genome and protein sequences of Ae. sharonensis and Ae. speltoides were obtained from PGSB PlantsDB ${ }^{3}$. A blastp search was performed against the protein sequences of the above species using the HSP90 sequences of Arabidopsis and rice as queries with the following parameters: an e-value lower than 1e-5 and an identity score above $50 \%$. Using the HMM profiles of the HSP90 domain (PF00183) downloaded from the Pfam database, the Hmmsearch engine in the HMMER3.0 program was also used to search these proteins with a threshold of 1e-5. Then, the blastp and HMMER results were merged, and redundancy was removed. All of the obtained HSP90 candidate sequences were subjected to search against the SMART database ${ }^{4}$ (Letunic and Bork, 2018) to manually confirm the presence of the histidine kinase-like ATPases (HATPase_c) and HSP90 domains (Zhang et al., 2013; Agarwal et al., 2016). Candidates that contained both the HATPase_c and HSP90 domain were regarded as HSP90s.

\section{Phylogenetic Relationship Analysis}

Multiple sequence alignment was performed by MAFFT (L-INSI algorithm) program using the protein sequences. Sequences from Arabidopsis and rice were used as markers to clarify the phylogeny. To investigate the evolutionary relationship of HSP90, a maximum likelihood tree was constructed using IQ-TREE (Nguyen et al., 2015). The substitution model was calculated with ModelFinder (integrated in IQ-TREE; best-fit model: JTT + G4 chosen according to the Bayesian information criterion) (Kalyaanamoorthy et al., 2017). The phylogenetic tree was examined by Ultrafast bootstraps (with parameters "-bb 1,000 -bnni") as well as a Shimodaira-Hasegawa approximate likelihood ratio test (SH-aLRT, with parameters "-alrt 1,000") (Guindon et al., 2010; Minh et al., 2013; Hoang et al., 2018). The tree file was visualized by Interactive Tree Of Life (iTOL) $\mathrm{v} 4^{5}$ (Letunic and Bork, 2019).

\section{Naming of Hexaploid Wheat HSP90s}

For clarity, we renamed all the HSP90s of hexaploid wheat, taking into account the naming convention of HSP 90 family members in Arabidopsis and rice. Each gene name started with the abbreviation of the hexaploid wheat (T. aestivum, Ta), followed by the abbreviation of this gene family (HSP90) and the subfamily name that is in Arabidopsis (AA, AB, B, C1, and C2), and finally ended with the chromosome and subgenome information. For example, the name of gene with geneID "TraesCS2A01G033700"

\footnotetext{
${ }^{1}$ https://urgi.versailles.inra.fr/

${ }^{2}$ http://plants.ensembl.org/

${ }^{3} \mathrm{ftp} / / / \mathrm{ftpmips}$.helmholtz-muenchen.de/plants/

${ }^{4} \mathrm{http}: / /$ smart.embl-heidelberg.de/

${ }^{5}$ https://itol.embl.de/
}

was renamed as TaHSP90AA-2A; in other words, it was a HSP90 gene belonging to the subfamily AA, and it was located on the AA subgenome on chromosome group 2. HSP90s from progenitors of hexaploid wheat were only presented in the phylogenetic relationship analysis; thus, they were not renamed.

\section{Transcriptional Regulation and AS Analysis of TaHSP90s}

The transcriptome data were obtained from our previous research (Wang et al., 2019). Briefly, hexaploid wheat plants (T. aestivum cv. Chinese Spring) were grown in greenhouse under normal condition. After 15 days from anthesis, the plants were subjected to heat stress $\left(37 / 17^{\circ} \mathrm{C}, 14 / 10 \mathrm{~h}\right)$. The filling grains and flag leaves were sampled at different time points and then for RNA isolation. The RNA-seq data, generated by Illumina (150 bp paired-end sequencing by Illuminas HiSeq X Ten platform) and PacBio (PacBio RS II platform) sequencing, and data processing, isoform characterization were performed as described in a previous study (Wang et al., 2019). Briefly, the PacBio sequencing full-length and non-chimeric (FLNC) reads were first mapped to the bread wheat reference genome, and the mapped FLNC reads were filtered and corrected with our previously defined criterion. Then, the FLNC reads that were mapped to the same genome loci and shared the same splicing junctions were collapsed into one isoform. Finally, the identified isoforms were further filtered based on the number of FLNC reads supporting this isoform, percentageof-identity of FLNC read-genome alignments, and the junction site verifications, whether they were supported by Illumina reads or genomic annotations as our previous description. The abundances of genes and isoforms were calculated by fragments per kilobase of exon per million fragment mapped (FPKM) values. The heat maps were drawn by "pheatmap" package in R software (version 3.6.1) with log2-transformed $($ FPKM +1$)$ values (Kolde and Kolde, 2015). Differentially expressed genes were identified by EdgeR (Robinson et al., 2010), and the genes that displayed fold change $\geq 2$ and an FDR adjusted $P$-value $<0.01$ were defined as differentially expressed genes, with non-stressed samples as the control. The gene with either of the following situation was regarded as a differentially spliced gene: (i) isoform set (FPKM $\geq 1$ and fully supported by Illumina reads) differed between heat stress and control sample. (ii) The isoform expression percentage (IEP) changed by more than $30 \%$ between the control and heat stress samples (Wang et al., 2019).

\section{RESULTS}

\section{HSP90 Genes in Hexaploid Wheat and Its Progenitors}

As a result of sequence searches and domain confirmation, 5, $5,4,5,11,13$, and 18 HSP90s were identified in T. urartu (AA progenitor), Ae. sharonensis (possible $\mathrm{BB}$ progenitor), Ae. speltoides (possible $\mathrm{BB}$ progenitor), Ae. tauschii (DD progenitor), wild emmer wheat, durum wheat, and hexaploid 


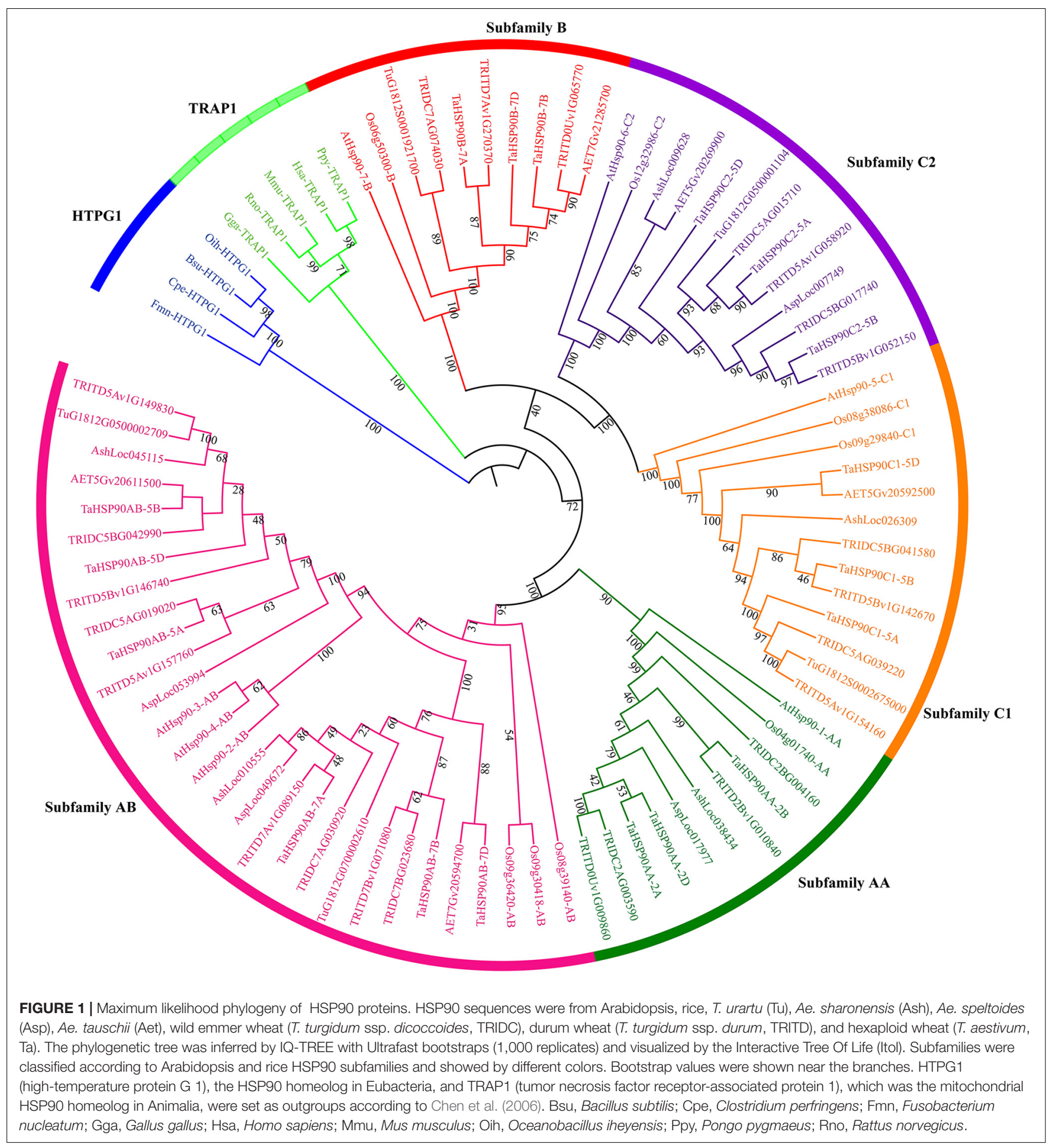

wheat genome, respectively. According to the phylogenetic tree, these HSP90s were clearly classified into subfamily AA, AB, B, $\mathrm{C} 1$, and $\mathrm{C} 2$ referred to as the classification of Arabidopsis and rice (Figure 1).

The clear polyploidization process of hexaploid wheat provided an opportunity to study the evolution of HSP90s during this process (Figure 2A) (Dubcovsky and Dvorak, 2007; Marcussen et al., 2014). The numbers of HSP90s were around five for the diploid species, and the number in allotetraploid wild emmer wheat (11) seemed to be the sum of the two diploid progenitors. However, the number of HSP90s in hexaploid wheat (18) was somehow not the sum of the allotetraploid wild emmer wheat (11) and the diploid Ae. tauschii (5) (Figure 2). For example, the hexaploid and the allotetraploid wheat contain three and two HSP90AA members, respectively, but the HSP90AA members were absent in diploid progenitor 


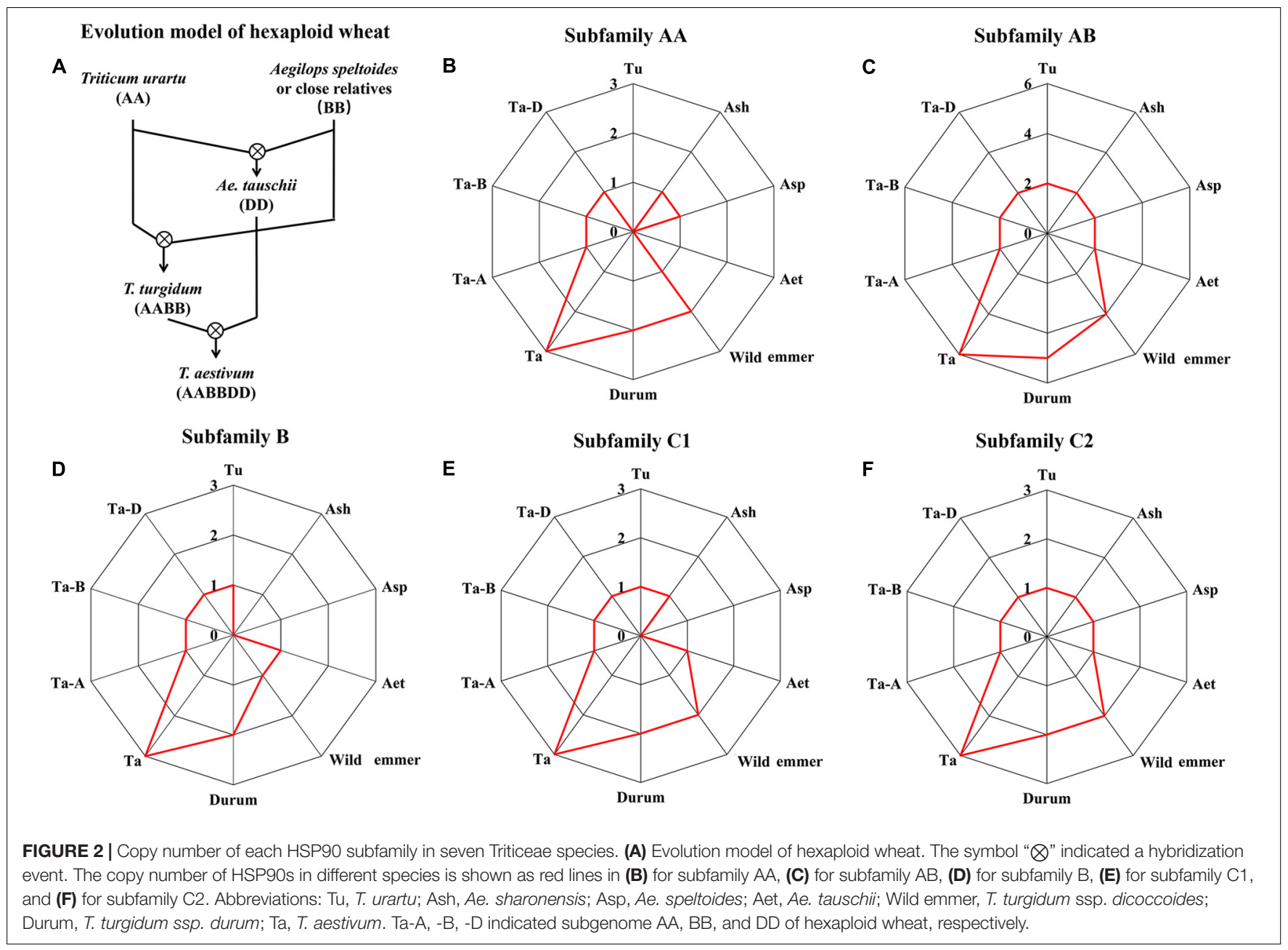

T. urartu and Ae. tauschii genomes. Syntenic analysis showed that the genomic segment containing the HSP90AA members and other three neighborhood genes were absent in the two diploid genomes (Supplementary Figure 1), suggesting hexaploid, and the allotetraploid wheat may acquire the gene copy during polyploidization, or the diploid genomes were not completely assembled. Conclusively, the copy number variation of HSP90 was not completely consistent with the polyploidy level.

\section{TaHSP90s Are Highly Conserved Between Three Homeologs}

The 18 identified HSP90s in hexaploid wheat (TaHSP90s) were present in homeologous chromosome groups 2, 5, and 7 and were classified into TaHSP90AAs (3), TaHSP90ABs (6), TaHSP90Bs (3), TaHSP90C1s (3), and TaHSP90C2s (3) based on the phylogenetic tree (Figure 1 and Table 1). Intriguingly, the TaHSP90s in each subfamily were evenly distributed among the AA, BB, and DD subgenomes, and the three TaHSP90s on the same chromosome group in each subfamily were regarded as the three TaHSP90 homeologs here after.

Sequence analysis showed that the protein sequence identities of the three TaHSP90 homeologs were above 96\%
(Supplementary Table 1). Accordingly, the protein sequence motifs (Supplementary Figure 2A and Supplementary Table 2) and gene structures (Supplementary Figure 2B) were also highly consistent among three TaHSP90 homeologs. Particularly, all the three TaHSP90 homeologs contained the same exon number, and that is $3,3,15,19$, and 20 for subfamily AA, $\mathrm{AB}, \mathrm{B}, \mathrm{C} 1$, and $\mathrm{C} 2$, respectively. These results showed that the sequences and gene structures are highly conserved within three TaHSP90s homeologs in hexaploid wheat, which were consistent with the reports in Populus and Brachypodium distachyon (Zhang et al., 2013, 2017).

\section{Conserved Heat Response Pattern Among Three TaHSP9O Homeologs}

Using the dynamic and intensive heat response transcriptomes of filling grains and flag leaves of wheat generated by our previous study (Wang et al., 2019), we investigated the heat response patterns of TaHSP90s and found that the heat stress response trend of each member of three TaHSP9O homeologs was largely conserved, although the expression abundance was slightly different (Figure 3A and Supplementary Figure 3). For example, all the three TaHSP90AAs homeologs were lowly expressed under 
TABLE 1 | Summary information of TaHSP90s.

\begin{tabular}{|c|c|c|c|c|c|}
\hline \multirow[t]{2}{*}{ TaHSP90s } & \multirow[t]{2}{*}{ GenelD } & \multirow[t]{2}{*}{ Exon number } & \multirow[t]{2}{*}{ Isoform number } & \multicolumn{2}{|c|}{ FPKM values } \\
\hline & & & & Grains & Flag leaves \\
\hline TaHSP90AA-2A & TraesCS2A01G033700 & 3 & 5 & $22-363$ & $2-1,896$ \\
\hline TaHSP90AA-2B & TraesCS2B01G047400 & 3 & 6 & $9-275$ & $2-1,486$ \\
\hline TaHSP90AA-2D & TraesCS2D01G033200 & 3 & 8 & $50-541$ & $18-2,496$ \\
\hline TaHSP90AB-7A & TraesCS7A01G242200 & 3 & 10 & $61-445$ & $148-2,249$ \\
\hline TaHSP90AB-7B & TraesCS7B01G149200 & 3 & 13 & $163-555$ & $160-2,045$ \\
\hline TaHSP9OAB-7D & TraesCS7D01G241100 & 3 & 6 & $83-378$ & $94-1,361$ \\
\hline TaHSP9OAB-5A & TraesCS5A01G260600 & 3 & 6 & $159-241$ & $141-350$ \\
\hline TaHSP9OAB-5B & TraesCS5B01G258900 & 3 & 9 & $79-181$ & $88-589$ \\
\hline TaHSP9OAB-5D & TraesCS5D01G268000 & 3 & 7 & $91-222$ & $86-632$ \\
\hline TaHSP9OB-7A & TraesCS7A01G529900 & 15 & 7 & $19-59$ & $67-232$ \\
\hline TaHSP9OB-7B & TraesCS7B01G446900 & 15 & 13 & $24-66$ & $65-203$ \\
\hline TaHSP9OB-7D & TraesCS7D01G517800 & 15 & 7 & $20-78$ & $65-235$ \\
\hline TaHSP90C1-5A & TraesCS5A01G251000 & 19 & 2 & $32-89$ & $29-190$ \\
\hline TaHSP90C1-5B & TraesCS5B01G249000 & 19 & 4 & $22-119$ & $77-422$ \\
\hline TaHSP90C1-5D & TraesCS5D01G258900 & 19 & 2 & $15-99$ & $27-220$ \\
\hline TaHSP90C2-5A & TraesCS5A01G101900 & 20 & 5 & $5-12$ & $4-24$ \\
\hline TaHSP90C2-5B & TraesCS5B01G106300 & 20 & 9 & $6-12$ & $19-104$ \\
\hline TaHSP90C2-5D & TraesCS5D01G113700 & 20 & 7 & $7-13$ & $11-56$ \\
\hline
\end{tabular}

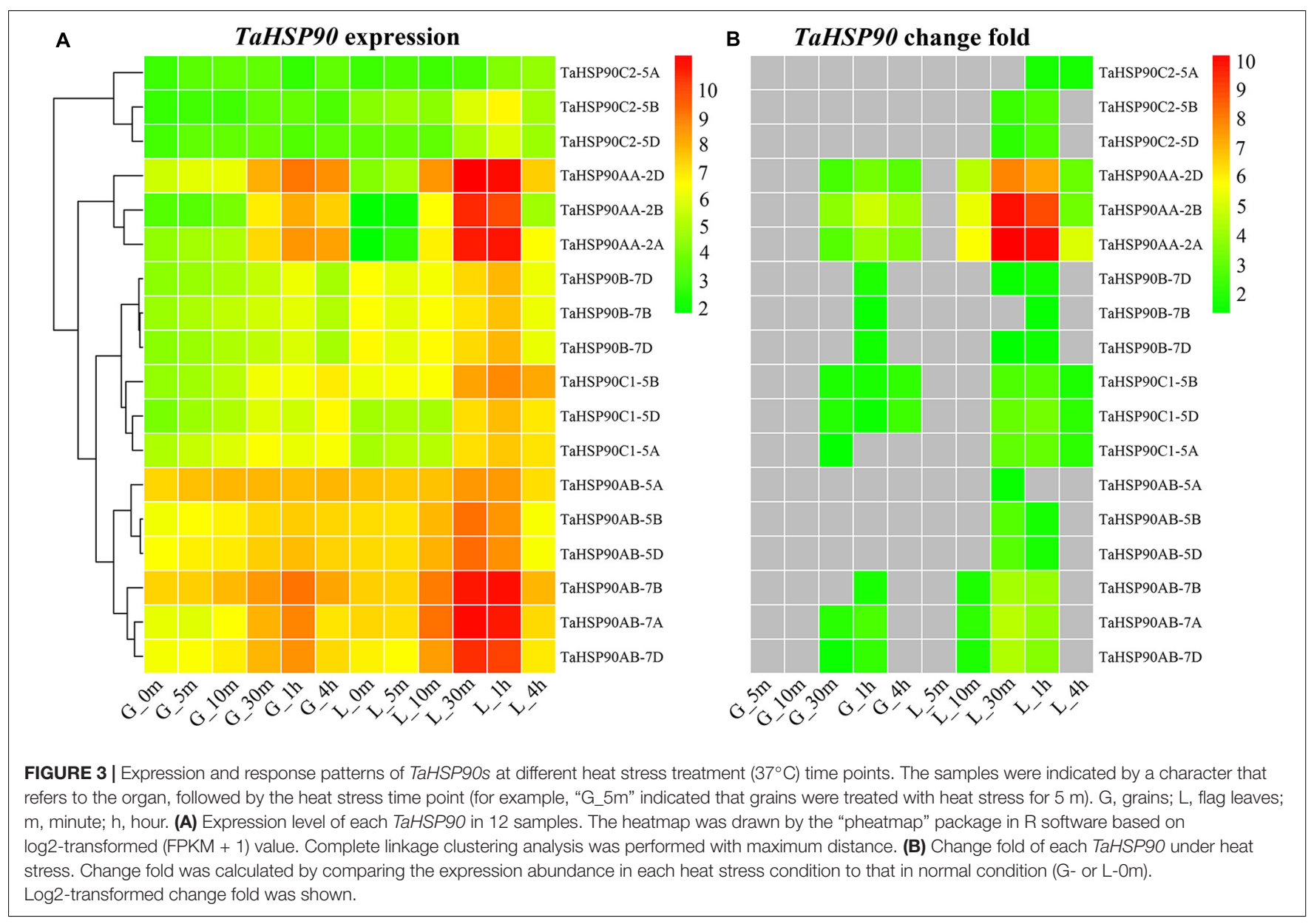




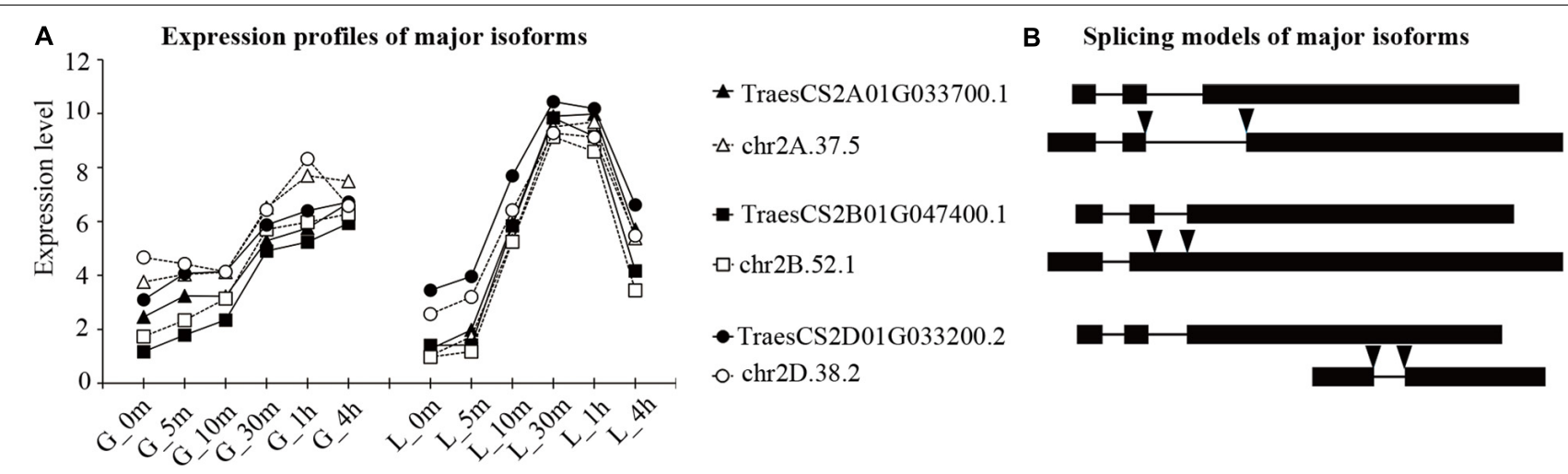

FIGURE 4 | Expression profiles and splicing modes of major isoforms generated by three TaHSP90AA homeologs. Isoform names are shown for both (A) and (B) (A) Expression profiles of major isoforms of TaHSP90AAs. Expression level is shown as the log2-transformed (FPKM +1$)$ value. The samples are indicated by a character that refers to the organ, followed by the heat stress duration (for example, "G_5m" indicated that grains were treated with heat stress for 5 m). G, grains; $L$, flag leaves; m, minute; h, hour. (B) Splicing modes of the major isoforms of TaHSP90AAs. Solid boxes represented exons, lines represented introns, and arrows indicated the splicing sites that differed from the longest intact coding sequence.

normal conditions in grains and flag leaves, and they were sharply upregulated (fold change $\geq 2$ and FDR-adjusted $P$-value $<0.01$ ) at 10 and $30 \mathrm{~min}$ heat stress treatment point in flag leaves, and grains, respectively (Figure 3B), consistent with HSP90AAs that were highly heat inducible (Swindell et al., 2007; Hu et al., 2009). Besides, all of the 18 TaHSP90s were heat responsive in flag leaves; however, it is also worth noting that all TaHSP90C2s (TaHSP90C2-5A, TaHSP90C2-5B, and TaHSP90C2-5D) and three TaHSP90ABs homeologs (TaHSP90AB-5A, TaHSP90AB-5B, and TaHSP90AB-5D) did not respond to heat stress in grains, suggesting a distinct response network between these two organs. In conclusion, together with the high level of sequence and gene structure conservation, these results demonstrated that no significant divergence of the three TaHSP90 homeologs occurred at transcriptional level in hexaploid wheat, though the heat response patterns of TaHSP90s were highly dynamic between different heat durations, gene subfamilies, and organs.

\section{Large Number of Novel Isoforms Generated by TaHSP90s Under Heat Stress}

Recent findings have suggested the importance of AS regulation in abiotic stress response (Reddy et al., 2013; Jiang et al., 2017; Keller et al., 2017; Laloum et al., 2018; Liu et al., 2018). Using the qualitative and quantitative heat response transcriptomes of filling grains and flag leaves produced by combining second- and third-generation sequencing in our previous study (Wang et al., 2019), we comprehensively investigated the roles of AS in the heat response of TaHSP90s.

First, a total of 126 isoforms of TaHSP90s were identified from our data, including the 36 isoforms that had been annotated in IWGSC RefSeq v1.0 and 90 newly identified isoforms (Supplementary Tables 3, 4 and Supplementary Figures 4, 5). The number of isoforms per TaHSP90 gene ranged from 2 to 13, with an average of 9.0, 8.5, 7.0, 6.3, and 2.7 for the subfamily $\mathrm{B}, \mathrm{AB}, \mathrm{C} 2, \mathrm{AA}$, and $\mathrm{C} 1$, respectively (Table 1). Although the exon-intron structures were highly conserved among the three TaHSP90 homeologs (Supplementary Figure 2B), the isoform numbers generated by the three TaHSP90 homeologs were such distinct. The most distinct change was observed in the three TaHSP90AB homeologs on chromosome group 7; the isoform numbers ranged from 6 to 13. In this case, the highly conserved TaHSP90s homeologs would possibly diverge at AS level by generating different isoform numbers under heat stress.

Second, with the quantified information of each isoform, we found that the six TaHSP90s (TaHSP90AB-5A, TaHSP90AB5B, TaHSP90AB-5D, TaHSP90C2-5A, TaHSP90C2-5B, and TaHSP90C2-5D) that were not heat responsive in grains generated some isoforms that responded to heat stress with transcriptional regulation (fold change $\geq 2$ and FDR-adjusted $P$-value $<$ 0.01) (Supplementary Figure 6). Thus, these transcriptionally heat-responsive isoforms extended our understanding of the transcriptional regulation of TaHSP90s and further revealed the complexity of the heat stress response for this gene family.

Next, to characterize the predominant isoforms of each TaHSP90 gene that may play more important roles, we introduced the IEP, which was calculated as the expression abundance ratio of one isoform to all isoforms generated by the same gene. An isoform with an average IEP of more than $30 \%$ across all of the time points in an organ was regarded as a major isoform, an isoform with an IEP less than 5\% in all time points was regarded as a rare isoform, and all other isoforms were classified as minor isoforms. This analysis led to the classification of isoforms into major (30), minor (44), and rare (52) isoforms (Supplementary Table 5). For 18 TaHSP90s, one TaHSP90 (TaHSP90AB-5B) generated three major isoforms, 12 TaHSP90s generated two major isoforms, and five TaHSP90s generated one major isoform. Interestingly, among the two or three major isoforms, one was already annotated in IWGSC RefSeq v1.0 and contained the longest complete coding region, which potentially encoded a functional peptide containing both the HATPase domain and HSP90 domain. However, another 

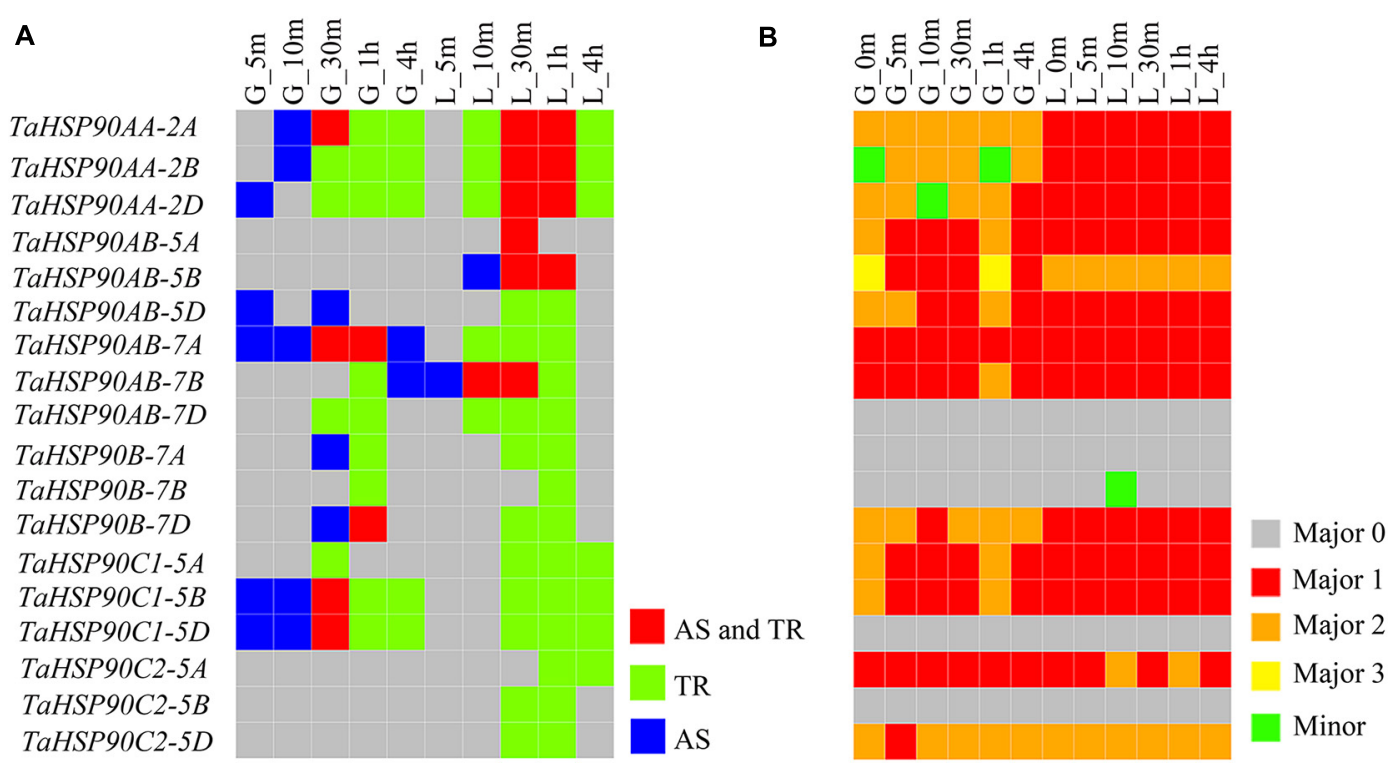

FIGURE 5 | Differential AS response of TaHSP90s under heat stress. The samples are indicated by a character that refers to the organ, followed by the heat stress duration (for example, "G_5m" indicated that grains were treated with heat stress for 5 m). G, grains; L, flag leaves; m, minute; h, hour. (A) Responses of TaHSP90s at different levels under different conditions. AS, alternative splicing response. TR, transcriptional response. (B) The most abundant isoform of each TaHSP90 in each sample. For the five TaHSP90s (TaHSP90AB-7D, TaHSP90B-7A, TaHSP90B-7B, TaHSP90C1-5D, TaHSP90C2-5B), they only generated one major isoform (Major 0). For the other TaHSP90s, which generated more than one major isoform; Major 1 represented the major isoform with the complete reading frame, and Major 2 and Major 3 (for TaHSP9OAB-5B only) represented the other major isoforms. Minor represented the minor isoform.

major isoform was newly discovered from our hybrid sequencing data, and this possessed only the HSP90 domain that potentially encoded truncated peptides. Furthermore, expression analysis showed that the major isoforms generated by the same gene had comparable expression levels and response patterns (Figure 4 and Supplementary Figures 7, 8), making it intriguing as to what roles these newly discovered isoforms played in the heat stress response.

\section{Varied Number and Splicing Modes of Major Isoforms Generated by TaHSP90 Homeologs}

The above transcription analysis showed that the expression abundance and response patterns of three TaHSP90 homeologs were conserved. However, regarding the number of major isoforms generated by three TaHSP90 homeologs, only one or two homeologs generated two major isoforms in subfamily $A B$, $\mathrm{B}, \mathrm{C} 1$, and $\mathrm{C} 2$, with the exception of subfamily AA in which each TaHSP90 homeolog generated two major isoforms (Figure 4 and Supplementary Figures 7, 8), demonstrating a varied major isoform number among three TaHSP90 homeologs.

Furthermore, the newly identified major isoforms (NIMIs) from our hybrid sequencing data also exhibited different exon and intron compositions among the three TaHSP90 homeologs, although they had the same gene structure, implying that the three TaHSP90 homeologs may exploit different peptides to respond to heat stress. For example, among the three TaHSP90AAs homeologs, the NIMI of TaHSP90AA-2A underwent AS at both the $5^{\prime}$ (Alt5'SS) and the $3^{\prime}$ ends of the first intron (Alt3'SS). The NIMI of TaHSP90AA-2B underwent intron retention. The NIMI of TaHSP90AA-2D underwent exon skipping (Figure 4). In conclusion, the number and isoform structure generated by the three TaHSP90 homeologs were significantly different, despite the three TaHSP90 homeologs being conserved at the sequence level and at the transcriptional response level. The differential number and isoform structure possibly suggested a new direction for evolutionary divergence of TaHSP90 homeologs in hexaploid wheat.

\section{Differential AS Responses of Three TaHSP90 Homeologs}

Comparing the control and heat stress treatment samples, we identified 12 TaHSP90s, which responded to heat stress by generating new isoforms or changing the expression level of highly expressed isoforms with the criterion defined in our previous study (Figure 5A) (Wang et al., 2019). Interestingly, some TaHSP90s that did not respond to heat stress with transcriptional regulation did respond to heat stress with AS regulation, particularly in grains under short time (5 and $10 \mathrm{~min}$ ) heat stress, extending our understanding of the heat response of TaHSP90s. Significantly, for the three TaHSP90 homeologs in subfamily $\mathrm{B}, \mathrm{C} 1$, and $\mathrm{AB}$ on chromosome group 7 , only one or two homeologs responded to heat stress with AS regulation, and in subfamily AA, though all three TaHSP90AAs underwent AS responses, but the AS responses occurred at different heat stress time points in grains. These results demonstrated the differential responses of three TaHSP90s homeologs at the AS regulation level. 
Furthermore, using the qualitative and quantitative isoforms, we investigated the isoform with the highest abundance for each TaHSP90 at each heat stress time point sample (Figure 5B). The highest abundance isoforms generated by three TaHSP90 homeologs at specific time point were also distinct, providing another evidence for the differential AS responses and suggesting possible diverged evolution. In conclusion, inconsistent with the conserved sequences and transcriptional regulation, AS response diverged among the three TaHSP90 homeologs, extending our understanding about the functional conservation and divergence of this gene family in hexaploid wheat.

\section{DISCUSSION}

HSP90s play vital roles in plant growth and stress response (Wang et al., 2004; Wang et al., 2016; Gil et al., 2017). In this study, we first performed a genome-wide analysis of HSP90s in hexaploid wheat and its progenitors. The copy numbers of HSP90s among these species were not consistent with polyploidy level. Next, we comprehensively analyzed the heat response patterns of TaHSP90s and found that AS diversified the heat response of TaHSP90s, suggesting different options for functional studies and breeding strategies. Meanwhile, our results provided a new perspective for understanding about evolutionary conservation and divergence for the homeologous genes in polyploidy species.

It has been reported that HSP90AAs were dramatically upregulated during heat stress, and HSP90ABs were constitutively expressed in Arabidopsis (Swindell et al., 2007) and rice ( $\mathrm{Hu}$ et al., 2009). However, with more intensive time course transcriptomes and different organs, we showed that all of the TaHSP90s were heat responsive under at least one time point in flag leaves, suggesting that this was a specific feature in hexaploid wheat or hinting that more investigations should be performed in Arabidopsis and rice to draw a conclusion. However, it was noteworthy that our data mainly focused on the short time heat response, and the TaHSP90s that do not respond to heat stress in grains may also respond to heat stress in other conditions.

The differentiation and subfunctionalization of homeologous genes are intensified by stress and are thought to contribute to the acclimation of polyploidy plants to stress (Dong and Adams, 2011; Liu et al., 2015; Powell et al., 2017). For example, about $68 \%$ of homeologous genes display expression partitioning according to the extent of stress responsiveness in hexaploid wheat (Liu et al., 2015). The homeolog-specific expression patterns of homeologous genes were also widely reported in wheat genes, resulting in different morphological phenotypes like lateral root number (Wang et al., 2018a), root hair length (Han et al., 2016), and other domestication traits (Zhang et al., 2011). For HSP90s, members of three TaHSP90C2 homeologs and three TaHSP90AB homeologs were also predicted to experience expression partitioning under drought stress and combined drought-heat stress, but not under only heat stress (Liu et al., 2015). Similarly, in our analysis, the heat expression trends and fold changes of TaHSP90 members were not significantly distinguishable between each other in three TaHSP90 homeologs. Thus, it seemed like the three
TaHSP90 homeologs were conserved and had not undergone subfunctionalization or neofunctionalization in heat response at transcriptional level, which was consistent with the highly conserved sequences and motifs.

However, in our subsequent analysis, we found that the numbers of major isoforms were distinct among three TaHSP90 homeologs, and further investigations revealed that the AS modes of the major isoforms generated by the three TaHSP90 homeologs were also not conserved, suggesting that the differentiation of TaHSP90 homeologs may occur at the AS level. Different splicing patterns have also been characterized among homeologous genes in allopolyploid cotton (Wang et al., 2018b). More than $51 \%$ of the homeologous genes generated isoforms containing different structure in allotetraploid cottons (Wang et al., 2018b). Theoretically, the differentiation in AS that resulted in distinct transcripts may lead to the diversified functions of homeologous genes (Long et al., 2013). Thus, divergent AS patterns and differential AS responses may contribute to the functional divergence and differential evolution of TaHSP90 homeologs, changing our understanding of the conservation of HSP90s in terms of expression profile and function.

In this study, TaHSP90s were found to generate many novel isoforms in the grain-filling stage under heat stress. Contrary to that, the expression of the abnormal isoforms was generally lower than those of the full-length isoforms of Lipoxygenase members in the tea plant in response to low temperature (Zhu et al., 2018), the expression levels of the truncated major isoforms and their full-length counterparts were found to be comparable in the current study. It was worthy to note that the peptides encoded by the truncated major isoforms only contain the HSP90 domain (Supplementary Table 3), making the roles of these truncated major isoforms intriguing. It is well known that the HATPase domain is responsible to bind ATP; the HSP90 domain is responsible for homodimerization and binding to clients (Schopf et al., 2017). We proposed that the truncated peptides modify their original functions by modulating the domain composition. For example, the novel major isoform of TaHSP90AA-2A possibly encoded a peptide containing the intact HSP90 domain but lost the HATPase domain. The truncated peptide may still form a homodimer but fail to bind clients without the ability to bind ATP; it seemed to decrease the protecting capacity of HSP90, and this was quite different to the AS regulation of HSFA2 under heat stress (Sugio et al., 2009; Liu et al., 2013; Cheng et al., 2015). In contrast, a total of 70 isoforms, including 36 novel isoforms, were annotated to comprise both the HATPase and HSP90 domain. By this way, these isoforms would probably encode different proteins and significantly increase HSP90 protein diversity to protect different substrates. The contrary hypothesis of the roles of the different isoforms should be elucidated in further studies. Furthermore, as HSP90s were found to translocate into the nucleus under heat stress, alternative TaHSP90 isoforms finally were transported into the nucleus would result in a different expression of different heat-responsive genes. Another question was how many isoforms could be finally translated into proteins, as isoforms arose from AS always subsequently translated into normal or truncated proteins, or degraded by the 
non-sense-mediated decay pathway (Kalyna et al., 2012; Syed et al., 2012; Chaudhary et al., 2019)?

In grains, the TaHSP90AAs, the major heat-responsive HSP90s, tended to favor the novel truncated major isoform and minor isoform in most samples; the other eight TaHSP90s also favored different isoforms in different samples. These changes raised questions whether they correlated to the delay heat response in grains or thermotolerance of grains. These results also remind us that when investigating the functions of homeologous genes, expressions and splicing types of isoforms would be important and worthy of study.

About $40 \%$ of the differentially spliced genes were also found to be regulated at the transcriptional level, inferring the vital role of the cooperation of AS and transcriptional regulation in heat response in hexaploid wheat (Liu et al., 2018). In the present study, all of the 18 TaHSP90s were transcriptionally regulated, and 12 of these were also AS regulated. The higher cooperation ratio of $\mathrm{AS}$ and transcriptional regulation may contribute to fine modulation of TaHSP90s, to match its key roles in heat stress response.

\section{DATA AVAILABILITY STATEMENT}

The datasets presented in this study can be found in online repositories. The names of the repository/repositories and accession number(s) can be found below: https://www.ncbi.nlm. nih.gov/sra/SRP128236.

\section{REFERENCES}

Agarwal, G., Garg, V., Kudapa, H., Doddamani, D., Pazhamala, L. T., Khan, A. W., et al. (2016). Genome-wide dissection of AP2/ERF and HSP90 gene families in five legumes and expression profiles in chickpea and pigeonpea. Plant Biotechnol. J. 14, 1563-1577. doi: 10.1111/pbi.12520

Asseng, S., Ewert, F., Martre, P., Rötter, R. P., Lobell, D. B., Cammarano, D., et al. (2015). Rising temperatures reduce global wheat production. Nat. Clim. Change 5:143.

Borrill, P., Adamski, N., and Uauy, C. (2015). Genomics as the key to unlocking the polyploid potential of wheat. New Phytol. 208, 1008-1022. doi: 10.1111/ nph.13533

Carretero-Paulet, L., Albert, V. A., and Fares, M. A. (2013). Molecular evolutionary mechanisms driving functional diversification of the HSP90A family of heat shock proteins in eukaryotes. Mol. Biol. Evol. 30, 2035-2043. doi: 10.1093/ molbev/mst113

Chaudhary, S., Jabre, I., Reddy, A. S. N., Staiger, D., and Syed, N. H. (2019). Perspective on alternative splicing and proteome complexity in plants. Trends Plant Sci. 24, 496-506. doi: 10.1016/j.tplants.2019.02.006

Chen, B., Zhong, D., and Monteiro, A. (2006). Comparative genomics and evolution of the HSP90 family of genes across all kingdoms of organisms. BMC Genom. 7:156. doi: 10.1186/1471-2164-7-156

Cheng, Q., Zhou, Y., Liu, Z., Zhang, L., Song, G., Guo, Z., et al. (2015). An alternatively spliced heat shock transcription factor, OsHSFA2dI, functions in the heat stress-induced unfolded protein response in rice. Plant Biol. 17, 419-429. doi: 10.1111/plb.12267

Dong, S., and Adams, K. L. (2011). Differential contributions to the transcriptome of duplicated genes in response to abiotic stresses in natural and synthetic polyploids. New Phytol. 190, 1045-1057. doi: 10.1111/j.1469-8137.2011. 03650.x

Dubcovsky, J., and Dvorak, J. (2007). Genome plasticity a key factor in the success of polyploid wheat under domestication. Science 316, 1862-1866. doi: 10.1126/ science. 1143986

\section{AUTHOR CONTRIBUTIONS}

SX and XW designed the study. YL, PZ, AZ, LM, SX, and XW analyzed the data. YL, SX, and XW wrote the manuscript. All authors contributed to the article and approved the submitted version.

\section{FUNDING}

This research was funded by the National Key Research and Development Program of China (2016YFD0101602), the National Natural Science Foundation of China (31501380), and the Open Project Program of the State Key Laboratory of Crop Stress Biology for Arid Areas (CSBAA2019005).

\section{ACKNOWLEDGMENTS}

This manuscript has been released as a pre-print at Research Square (Lu et al., 2019).

\section{SUPPLEMENTARY MATERIAL}

The Supplementary Material for this article can be found online at: https://www.frontiersin.org/articles/10.3389/fgene. 2020.577897/full\#supplementary-material

Geiler-Samerotte, K. A., Zhu, Y. O., Goulet, B. E., Hall, D. W., and Siegal, M. L. (2016). Selection transforms the landscape of genetic variation interacting with Hsp90. PLoS Biol. 14:e2000465. doi: 10.1371/journal.pbio.2000465

Gil, K.-E., Kim, W.-Y., Lee, H.-J., Faisal, M., Saquib, Q., Alatar, A. A., et al. (2017). ZEITLUPE contributes to a thermoresponsive protein quality control system in Arabidopsis. Plant Cell 29, 2882-2894. doi: 10.1105/tpc.17.00612

Guindon, S., Dufayard, J.-F., Lefort, V., Anisimova, M., Hordijk, W., and Gascuel, O. (2010). New algorithms and methods to estimate maximum-likelihood phylogenies: assessing the performance of PhyML 3.0. Systematic Biol. 59, 307-321. doi: 10.1093/sysbio/syq010

Hahn, A., Bublak, D., Schleiff, E., and Scharf, K.-D. (2011). Crosstalk between Hsp90 and Hsp70 chaperones and heat stress transcription factors in tomato. Plant Cell 23, 741-755. doi: 10.1105/tpc.110.076018

Han, Y., Xin, M., Huang, K., Xu, Y., Liu, Z., Hu, Z., et al. (2016). Altered expression of TaRSL4 gene by genome interplay shapes root hair length in allopolyploid wheat. New Phytol. 209, 721-732. doi: 10.1111/nph. 13615

Hoang, D. T., Chernomor, O., Von Haeseler, A., Minh, B. Q., and Vinh, L. S. (2018). UFBoot2: improving the ultrafast bootstrap approximation. Mol. Biol. Evol. 35, 518-522. doi: 10.1093/molbev/msx281

Hu, W., Hu, G., and Han, B. (2009). Genome-wide survey and expression profiling of heat shock proteins and heat shock factors revealed overlapped and stress specific response under abiotic stresses in rice. Plant Sci. 176, 583-590. doi: 10.1016/j.plantsci.2009.01.016

International Wheat Genome Sequencing Consortium [IWGSC] (2018). Shifting the limits in wheat research and breeding using a fully annotated reference genome. Science 361:eaar7191. doi: 10.1126/science.aar7191

Jiang, J., Liu, X., Liu, C., Liu, G., Li, S., and Wang, L. (2017). Integrating omics and alternative splicing reveals insights into grape response to high temperature. Plant Physiol. 173, 1502-1518. doi: 10.1104/pp.16.01305

Kalyaanamoorthy, S., Minh, B. Q., Wong, T. K., von Haeseler, A., and Jermiin, L. S. (2017). ModelFinder: fast model selection for accurate phylogenetic estimates. Nat. Methods 14:587. doi: 10.1038/nmeth.4285 
Kalyna, M., Simpson, C. G., Syed, N. H., Lewandowska, D., Marquez, Y., Kusenda, B., et al. (2012). Alternative splicing and nonsense-mediated decay modulate expression of important regulatory genes in Arabidopsis. Nucleic Acids Res. 40, 2454-2469. doi: 10.1093/nar/gkr932

Keller, M., Hu, Y., Mesihovic, A., Fragkostefanakis, S., Schleiff, E., and Simm, S. (2017). Alternative splicing in tomato pollen in response to heat stress. DNA Res. 24, 205-217.

Kolde, R., and Kolde, M. R. (2015). Package 'Pheatmap'. http://cran.r-project.org/ web/packages/pheatmap/index.html (accessed March 12, 2020).

Krishna, P., and Gloor, G. (2001). The Hsp90 family of proteins in Arabidopsis thaliana. Cell Stress Chaperones 6:238. doi: 10.1379/1466-1268(2001)006<0238: thfopi $>2.0 . \operatorname{co} ; 2$

Laloum, T., Martín, G., and Duque, P. (2018). Alternative splicing control of abiotic stress responses. Trends Plant Sci. 23, 140-150. doi: 10.1016/j.tplants.2017.09. 019

Leach, L. J., Belfield, E. J., Jiang, C., Brown, C., Mithani, A., and Harberd, N. P. (2014). Patterns of homoeologous gene expression shown by RNA sequencing in hexaploid bread wheat. BMC Genom. 15:276. doi: 10.1186/1471-2164-15276

Lesk, C., Rowhani, P., and Ramankutty, N. (2016). Influence of extreme weather disasters on global crop production. Nature 529, 84-87. doi: 10.1038/ nature 16467

Letunic, I., and Bork, P. (2018). 20 years of the SMART protein domain annotation resource. Nucleic Acids Res. 46, D493-D496.

Letunic, I., and Bork, P. (2019). Interactive Tree Of Life (iTOL) v4: recent updates and new developments. Nucleic Acids Res. 47, W256-W259.

Liu, B., Liu, L., Tian, L., Cao, W., Zhu, Y., and Asseng, S. (2014). Post-heading heat stress and yield impact in winter wheat of China. Global Change Biol. 20, 372-381. doi: 10.1111/gcb.12442

Liu, J., Sun, N., Liu, M., Liu, J., Du, B., Wang, X., et al. (2013). An autoregulatory loop controlling Arabidopsis HsfA2 expression: role of heat shock-induced alternative splicing. Plant Physiol. 162, 512-521. doi: 10.1104/pp.112.205864

Liu, Z., Qin, J., Tian, X., Xu, S., Wang, Y., Li, H., et al. (2018). Global profiling of alternative splicing landscape responsive to drought, heat and their combination in wheat (Triticum aestivum L.). Plant Biotechnol. J. 16, 714-726. doi: $10.1111 /$ pbi. 12822

Liu, Z., Xin, M., Qin, J., Peng, H., Ni, Z., Yao, Y., et al. (2015). Temporal transcriptome profiling reveals expression partitioning of homeologous genes contributing to heat and drought acclimation in wheat (Triticum aestivum L.). BMC Plant Biol. 15:152. doi: 10.1186/s12870-015-0511-8

Long, M., VanKuren, N. W., Chen, S., and Vibranovski, M. D. (2013). New gene evolution: little did we know. Ann. Rev. Genet. 47, 307-333. doi: 10.1146/ annurev-genet-111212-133301

Lu, Y., Zhao, P., Zhang, A., Ma, L., Xu, S., and Wang, X. (2019*). Alternative splicing diversifies the heat response and evolutionary strategy of conserved Heat Shock Protein 90 in bread wheat (Triticum aestivum L.). Res. Square doi: $10.21203 /$ rs.2.17636/v1

Marcussen, T., Sandve, S. R., Heier, L., Spannagl, M., Pfeifer, M., Jakobsen, K. S., et al. (2014). Ancient hybridizations among the ancestral genomes of bread wheat. Science 345:1250092.

Meiri, D., and Breiman, A. (2009). Arabidopsis ROF1 (FKBP62) modulates thermotolerance by interacting with HSP90.1 and affecting the accumulation of HsfA2-regulated sHSPs. Plant J. 59, 387-399. doi: 10.1111/j.1365-313x.2009. 03878.x

Minh, B. Q., Nguyen, M. A. T., and von Haeseler, A. (2013). Ultrafast approximation for phylogenetic bootstrap. Mol. Biol. Evol. 30, 1188-1195. doi: 10.1093/molbev/mst024

Nguyen, L.-T., Schmidt, H. A., Von Haeseler, A., and Minh, B. Q. (2015). IQ-TREE: a fast and effective stochastic algorithm for estimating maximum-likelihood phylogenies. Mol. Biol. Evol. 32, 268-274. doi: 10.1093/molbev/msu300

Ohama, N., Sato, H., Shinozaki, K., and Yamaguchi-Shinozaki, K. (2017). Transcriptional regulatory network of plant heat stress response. Trends Plant Sci. 22, 53-65. doi: 10.1016/j.tplants.2016.08.015

Powell, J. J., Fitzgerald, T. L., Stiller, J., Berkman, P. J., Gardiner, D. M., Manners, J. M., et al. (2017). The defence-associated transcriptome of hexaploid wheat displays homoeolog expression and induction bias. Plant Biotechnol. J. 15, 533-543. doi: 10.1111/pbi.12651
Prasad, P. V., and Djanaguiraman, M. (2014). Response of floret fertility and individual grain weight of wheat to high temperature stress: sensitive stages and thresholds for temperature and duration. Funct. Plant Biol. 41, 1261-1269. doi: $10.1071 /$ fp14061

Queitsch, C., Sangster, T. A., and Lindquist, S. (2002). Hsp90 as a capacitor of phenotypic variation. Nature 417, 618-624. doi: 10.1038/nature749

Ramírez-González, R., Borrill, P., Lang, D., Harrington, S., Brinton, J., Venturini, L., et al. (2018). The transcriptional landscape of polyploid wheat. Science 361:eaar6089.

Reddy, A. S., Marquez, Y., Kalyna, M., and Barta, A. (2013). Complexity of the alternative splicing landscape in plants. Plant Cell 25, 3657-3683. doi: 10.1105/ tpc.113.117523

Robinson, M. D., McCarthy, D. J., and Smyth, G. K. (2010). edgeR: a Bioconductor package for differential expression analysis of digital gene expression data. Bioinformatics 26, 139-140. doi: 10.1093/bioinformatics/btp616

Rutherford, S. L., and Lindquist, S. (1998). Hsp90 as a capacitor for morphological evolution. Nature 396, 336-342. doi: 10.1038/24550

Schopf, F. H., Biebl, M. M., and Buchner, J. (2017). The HSP90 chaperone machinery. Nat. Rev. Mol. Cell Biol. 18:345.

Song, Z., Pan, F., Yang, C., Jia, H., Jiang, H., He, F., et al. (2019). Genomewide identification and expression analysis of HSP90 gene family in Nicotiana tabacum. BMC Genet. 20:35. doi: 10.1186/s12863-019-0738-8

Sugio, A., Dreos, R., Aparicio, F., and Maule, A. J. (2009). The cytosolic protein response as a subcomponent of the wider heat shock response in Arabidopsis. Plant Cell 21, 642-654. doi: 10.1105/tpc.108.062596

Swindell, W. R., Huebner, M., and Weber, A. P. (2007). Transcriptional profiling of Arabidopsis heat shock proteins and transcription factors reveals extensive overlap between heat and non-heat stress response pathways. BMC Genom. 8:125. doi: 10.1186/1471-2164-8-125

Syed, N. H., Kalyna, M., Marquez, Y., Barta, A., and Brown, J. W. (2012). Alternative splicing in plants-coming of age. Trends Plant Sci. 17, 616-623. doi: 10.1016/j.tplants.2012.06.001

Wang, G. F., Wei, X., Fan, R., Zhou, H., Wang, X., Yu, C., et al. (2011). Molecular analysis of common wheat genes encoding three types of cytosolic heat shock protein 90 (Hsp90): functional involvement of cytosolic Hsp90s in the control of wheat seedling growth and disease resistance. New Phytol. 191, 418-431. doi: 10.1111/j.1469-8137.2011.03715.x

Wang, H., Hu, Z., Huang, K., Han, Y., Zhao, A., Han, H., et al. (2018a). Three genomes differentially contribute to the seedling lateral root number in allohexaploid wheat: evidence from phenotype evolution and gene expression. Plant J. 95, 976-987. doi: 10.1111/tpj.14005

Wang, M., Wang, P., Liang, F., Ye, Z., Li, J., Shen, C., et al. (2018b). A global survey of alternative splicing in allopolyploid cotton: landscape, complexity and regulation. New Phytol. 217, 163-178. doi: 10.1111/nph.14762

Wang, R., Zhang, Y., Kieffer, M., Yu, H., Kepinski, S., and Estelle, M. (2016). HSP90 regulates temperature-dependent seedling growth in Arabidopsis by stabilizing the auxin co-receptor F-box protein TIR1. Nat. Commun. 7:10269.

Wang, W., Vinocur, B., Shoseyov, O., and Altman, A. (2004). Role of plant heatshock proteins and molecular chaperones in the abiotic stress response. Trends Plant Sci. 9, 244-252. doi: 10.1016/j.tplants.2004.03.006

Wang, X., Chen, S., Shi, X., Liu, D., Zhao, P., Lu, Y., et al. (2019). Hybrid sequencing reveals insight into heat sensing and signaling of bread wheat. Plant J. 98, 1015-1032. doi: 10.1111/tpj.14299

Xu, Z.-S., Li, Z.-Y., Chen, Y., Chen, M., Li, L.-C., and Ma, Y.-Z. (2012). Heat shock protein 90 in plants: molecular mechanisms and roles in stress responses. Int. J. Mol. Sci. 13, 15706-15723. doi: 10.3390/ijms131215706

Yabe, N., Takahashi, T., and Komeda, Y. (1994). Analysis of tissue-specific expression of Arabidopsis thaliana HSP90-family gene HSP81. Plant Cell Physiol. 35, 1207-1219. doi: 10.1093/oxfordjournals.pcp.a078715

Zhang, J., Li, J., Liu, B., Zhang, L., Chen, J., and Lu, M. (2013). Genomewide analysis of the Populus Hsp90 gene family reveals differential expression patterns, localization, and heat stress responses. BMC Genom. 14:532. doi: 10. 1186/1471-2164-14-532

Zhang, M., Shen, Z., Meng, G., Lu, Y., and Wang, Y. (2017). Genome-wide analysis of the Brachypodium distachyon (L.) P. Beauv. Hsp90 gene family reveals molecular evolution and expression profiling under drought and salt stresses. PLoS One 12:e0189187. doi: 10.1371/journal.pone.0189187 
Zhang, Y., Liu, Z., Khan, A. A., Lin, Q., Han, Y., Mu, P., et al. (2016). Expression partitioning of homeologs and tandem duplications contribute to salt tolerance in wheat (Triticum aestivum L.). Sci. Rep. 6:21476.

Zhang, Z., Belcram, H., Gornicki, P., Charles, M., Just, J., Huneau, C., et al. (2011). Duplication and partitioning in evolution and function of homoeologous Q loci governing domestication characters in polyploid wheat. Proc. Natl. Acad. Sci. U $S$ A. 108, 18737-18742. doi: 10.1073/pnas.1110552108

Zhao, C., Liu, B., Piao, S., Wang, X., Lobell, D. B., Huang, Y., et al. (2017). Temperature increase reduces global yields of major crops in four independent estimates. Proc. Natl. Acad. Sci. U S A. 114, 9326-9331. doi: 10.1073/pnas. 1701762114

Zhu, J., Wang, X., Guo, L., Xu, Q., Zhao, S., Li, F., et al. (2018). Characterization and alternative splicing profiles of the Lipoxygenase gene family in tea plant (Camellia sinensis). Plant Physiol. 59, 1765-1781. doi: 10.1093/pcp/ pcy091

Conflict of Interest: The authors declare that the research was conducted in the absence of any commercial or financial relationships that could be construed as a potential conflict of interest.

Copyright (C) $2020 \mathrm{Lu}$, Zhao, Zhang, Ma, Xu and Wang. This is an open-access article distributed under the terms of the Creative Commons Attribution License (CC BY). The use, distribution or reproduction in other forums is permitted, provided the original author(s) and the copyright owner(s) are credited and that the original publication in this journal is cited, in accordance with accepted academic practice. No use, distribution or reproduction is permitted which does not comply with these terms. 